\title{
Situação dos medicamentos biológicos no Brasil
}

\section{Situation of biological medicines in Brazil}

DOI: $10.46814 /$ lajdv2n6-008

Recebimento dos originais: 01/09/2020

Aceitação para publicação: 30/10/2020

\section{Gabriel Duarte Mendonça}

Farmacêutico Generalista pela Universidade Federal do Rio de Janeiro (UFRJ)/Faculdade de Farmácia

Instituição: Faculdade de Farmácia da Universidade Federal do Rio de Janeiro

Endereço: Av. Carlos Chagas Filho 373, Ilha do Fundão; Rio de Janeiro - RJ, Brasil

E-mail: gabriel_mendonca_tp@hotmail.com

\section{Márcia Maria Barros dos Passos \\ Doutorado em Saúde Coletiva pela Universidade Estadual do Rio de Janeiro (UERJ)/Instituto de Medicina Social \\ Instituição: Faculdade de Farmácia da Universidade Federal do Rio de Janeiro Endereço: Av. Carlos Chagas Filho 373, Ilha do Fundão; Rio de Janeiro - RJ, Brasil \\ E-mail: mmbpassos@gmail.com}

\section{Ângelo Samir Melim Miguel}

Doutorado em Tecnologia Bioquímico-Farmacêutica pela Universidade de São Paulo (USP)

Instituição: Faculdade de Farmácia da Universidade Federal do Rio de Janeiro Endereço: Av. Carlos Chagas Filho 373, Ilha do Fundão; Rio de Janeiro - RJ, Brasil

E-mail: samir.ff.ufrj@gmail.com

\section{Theo Luiz Ferraz de Souza}

Doutorado em Química Biológica pela Universidade Federal Rio de Janeiro (UFRJ) Instituição: Faculdade de Farmácia da Universidade Federal do Rio de Janeiro Endereço: Av. Carlos Chagas Filho 373, Ilha do Fundão; Rio de Janeiro - RJ, Brasil

E-mail: theo.ff.ufrj@gmail.com

\section{RESUMO}

Medicamento biológico é aquele que possui princípio ativo de origem biológica com atividade terapêutica conhecida. Esta classe de produtos inclui várias categorias de medicamentos ordenados de acordo com a sua tecnologia de obtenção. São medicamentos largamente utilizados no país e constituem um mercado em potencial expansão. O objetivo deste trabalho foi analisar a situação dos medicamentos biológicos no Brasil, no contexto da Assistência Farmacêutica. Trata-se de um estudo descritivo exploratório a partir da busca de informações na Relação Nacional de Medicamentos Essenciais (RENAME), nas bases de dados: Scielo, Periódico capes, Visalegis e website do Instituto Nacional da Propriedade Intelectual (INPI), para consulta de patentes depositadas, e website da Agência Nacional de Vigilância Sanitária (Anvisa) para as regulamentações pertinentes ao registro de tais medicamentos. Os resultados indicaram a presença de 78 medicamentos biológicos (16\%) do total de medicamentos integrantes da RENAME 2017, que integram todos os Componentes da Assistência Farmacêutica: Básico, Estratégico e Especializado. Foi verificado que na sua maioria estes medicamentos são importados ou possuem apenas as etapas de embalagem pelas empresas 
nacionais. Adicionalmente, a presença ínfima de biossimilares foi observada, mesmo para aqueles medicamentos cuja patente já caiu em caducidade há muito tempo. Por fim, os dados apontam uma grande dependência tecnológica e produtiva do Brasil em relação ao mercado internacional de medicamentos biológicos, gerando um grande impacto financeiro, principalmente nos componentes estratégicos e no financiamento da Assistência Farmacêutica no Brasil.

Palavras Chaves: Medicamentos Biológicos, Assistência Farmacêutica, Política de Medicamentos, Biotecnologia, Indústria Farmacêutica.

\begin{abstract}
Biological medication is one that has an active ingredient of biological origin with known therapeutic activity. This product class includes several categories of medicines ordered according to their technology. They are medicines widely used in the country and constitute a potential expansion market. The objective of this work was to analyze the situation of biological medicines in Brazil, in the context of Pharmaceutical Assistance. This is an exploratory descriptive study based on the search for information in the National List of Essential Medicines (RENAME), in the databases: Scielo, Periodical capes, Visalegis and the website of the National Institute of Industrial Property (INPI), for consultation of patents deposited, and the website of the National Health Surveillance Agency (Anvisa) for regulations relevant to the registration of such drugs. The results indicated the presence of 78 biological medicines $(16 \%)$ of the total of medicines included in RENAME 2017, which integrate all the Pharmaceutical Assistance Components: Basic, Strategic and Specialized. It was found that most of these medicines are imported or have only the packaging steps by national companies. Additionally, the smallest presence of biosimilars was observed, even for those medicines whose patent has long since expired. Finally, the data points to a great technological and productive dependence of Brazil in relation to the international market of biological medicines, generating a great financial impact, mainly in the strategic components and in the financing of Pharmaceutical Assistance in Brazil.
\end{abstract}

Keywords: Biological Medicines, Pharmaceutical Assistance, Medicines Policy, Biotechnology, Pharmaceutical Industry.

\title{
1 INTRODUÇÃO
}

Os medicamentos biológicos são originados de fármacos exclusivamente de origem biológica. Geralmente são constituídos por moléculas complexas de alto peso molecular, obtidos a partir de fluidos biológicos, tecidos de origem animal ou procedimentos biotecnológicos por meio de manipulação ou inserção de outro material genético (tecnologia do DNA recombinante) ou alteração dos genes que ocorre devido à irradiação, produtos químicos ou seleção forçada (BRAGA, 2016).

Segundo a Resolução da Diretoria Colegiada - RDC nº 55, de 16 de dezembro de 2010, produto biológico é todo medicamento biológico que contenha molécula com atividade biológica conhecida, já registrado no Brasil e que tenha passado por todas as etapas de fabricação (formulação, envase, liofilização, rotulagem, embalagem, armazenamento, controle de qualidade e liberação do lote de produto biológico para uso). São classes de produtos biológicos listados nessa RDC: as 
vacinas; os soros hiperimunes; hemoderivados; os medicamentos biológicos obtidos a partir de fluidos biológicos ou de tecidos de origem animal e medicamentos obtidos por procedimento biotecnológico; os anticorpos monoclonais; e os medicamentos contendo micro-organismos vivos, atenuados ou mortos (BRASIL, 2010).

Os produtos biológicos apresentam condições de produção mais complexas do que os sintetizados quimicamente, por causa de sua heterogeneidade estrutural e interações dentro do sistema biológico. O processo de fabricação dos biológicos é muito mais elaborado já que varia de acordo com cada tipo de molécula devido ao uso de organismos vivos e substratos complexos (SAHOO et al, 2009). Requerem validação mais estrita e permanente avaliação do produto ao longo do processo de fabricação, já que há muito mais parâmetros que os afetam e por serem muito mais complexos. Enquanto estima-se que são realizados de 40 a 50 testes críticos de um produto químico, um produto biológico é testado 250 vezes ou mais durante o processo de manufatura para garantir a eficácia do medicamento (RAO, 2011).

Dessa forma, como se verifica, esses produtos apresentam grande diversidade e heterogeneidade quanto às tecnologias de obtenção, que impacta diretamente, nos custos da assistência farmacêutica, considerando principalmente aqueles que ainda estão sob patentes e exercem o monopólio na comercialização do produto, que sem competição no mercado, limita as estratégias para redução de preço por meio da aquisição de alternativas farmacêuticas (WHO, 2014).

No âmbito do Sistema Único de Saúde (SUS), de acordo com a Portaria GM/MS nº 204, de 29 de janeiro de 2007, as ações e os serviços de saúde estão organizados na forma de blocos de financiamento específicos, de acordo com seus objetivos e características. O bloco de financiamento para a Assistência Farmacêutica é constituído por três componentes: O Componente Básico da Assistência Farmacêutica está relacionado aos medicamentos e insumos da Assistência Farmacêutica no âmbito da Atenção Básica à Saúde; Componente Estratégico contempla medicamentos para o tratamento das doenças de perfil endêmico e que tenham impacto socioeconômico. Estes medicamentos têm controle e tratamento definidos por meio de protocolos e normas estabelecidas, e seu financiamento é de responsabilidade do Ministério da Saúde, que realiza sua aquisição e repasse aos estados ou municípios, de acordo com o planejamento anual. O componente Especializado da Assistência Farmacêutica (CEAF) contempla os tratamentos medicamentosos, em nível ambulatorial, cujas linhas de cuidado estão definidas e publicados nos Protocolos Clínicos e Diretrizes Terapêuticas (PCDT) do Ministério da Saúde, de acordo com os seguintes critérios: complexidade do tratamento da doença; garantia da integralidade do tratamento da doença no âmbito da linha de cuidado; e manutenção do equilíbrio financeiro entre as esferas de gestão do SUS. Estes 
medicamentos são medicamentos financiados pelo Ministério da Saúde e adquiridos pela Secretaria de saúde dos estados.

Neste contexto, o objetivo deste trabalho é descrever os medicamentos biológicos que integram a Relação Nacional de Medicamentos Essenciais (RENAME-2017) a qual componente da assistência farmacêutica pertence, e analisar sua situação quanto à registro, produção nacional e importação. Esta análise justifica-se pela relevância desta classe de medicamentos e o impacto que eles possuem na assistência farmacêutica, além de proporcionar análise quanto à dependência tecnológica internacional no que tange a pesquisa e desenvolvimento, propriedade intelectual, fabricação e comercialização.

\section{METODOLOGIA}

Trata-se de um estudo descritivo sobre a situação dos medicamentos biológicos disponíveis na Relação Nacional de Medicamentos Essenciais - RENAME 2017, que orienta a Assistência Farmacêutica (AF) no Brasil.

Foi realizada busca na literatura recente, por meio de consulta as bases eletrônicas como: Medline, PubMed, Scielo e Lilacs e Google Acadêmico, para construção do marco teórico e discussão dos resultados. A busca de referências nos periódicos utilizou os seguintes termos: produtos biológicos, medicamentos biológicos, legislação brasileira, vigilância sanitária, e também suas traduções correspondentes, em combinações alternadas.

Para a consulta do registro dos medicamentos na Anvisa, foi acessado o website <www.anvisa.gov.br> e seguido o caminho "Medicamentos", dentro de Assuntos, após "Medicamentos regularizados" dentro da opção "Consultas e Serviços", após "Medicamentos Registrados" e então eram inseridas alguma informação em "Critérios para consulta".

A escolha das palavras para pesquisa foi feita seguindo o nome do medicamento biológico presente na RENAME 2017, de acordo com a Denominação Comum Brasileira (DCB) e Denominação Comum Internacional (DCI).

Para a construção do banco de dados foram utilizadas as seguintes variáveis: medicamento, processo de obtenção, fabricante, fabricação nacional, fabricação internacional, presença na RENAME, Componente da assistência farmacêutica.

\section{RESULTADOS}


Foi verificada a presença de 78 medicamentos biológicos integrantes da RENAME 2017, que representam $16,5 \%$ do total de medicamentos presentes na lista de medicamentos essenciais que apresenta o conjunto de 472 medicamentos descritos em sua denominação genérica (BRASIL, 2017). Destes 3 integram o elenco de 179 medicamentos do Componente Básico, 53 do elenco de 150 do componente Estratégico, e 22 do elenco de 143 do componente Especializado .

Na tabela 1, são listados os medicamentos biológicos presentes na RENAME-2017, sua forma farmacêutica, a quantidade de empresas nacionais (FN) e de empresas internacionais (FI) que participam de alguma etapa da fabricação e, por fim, o componente da Assistência Farmacêutica (Comp. da AF) a que o medicamento pertence.

Tabela 1 - Perfil dos Medicamentos biológicos na RENAME-2017 quanto ao tipo de fabricante.

\begin{tabular}{|c|c|c|c|c|}
\hline Medicamentos biológicos na RENAME-2017 & $\mathbf{N}^{\mathbf{0}}$ Opções & FNI & FI & Componente \\
\hline Heparina sódica - $5.000 \mathrm{UI} / 0,25 \mathrm{~mL}$ & 1 & 1 & 0 & Básico \\
\hline Insulina humana NPH - $100 \mathrm{UI} / \mathrm{mL}$ & 3 & 0 & 4 & Básico \\
\hline Insulina humana regular - $100 \mathrm{UI} / \mathrm{mL}$ & 7 & 2 & 8 & Básico \\
\hline Complexo protrombínico humano - 500 UI a 600 UI & 3 & 1 & 9 & Estratégico \\
\hline Complexo protrombínico parcialmente ativado & 3 & 1 & 9 & Estratégico \\
\hline Fator IX de coagulação - 200 a 250 UI e 500 a 600 UI & 5 & 0 & 11 & Estratégico \\
\hline Fator VII ativado recombinante $-(1 \mathrm{mg}$ a $5 \mathrm{mg})$ & 1 & 0 & 2 & Estratégico \\
\hline Fator VIII associado ao Fator de von Willebrand. & 3 & 0 & 4 & Estratégico \\
\hline Fator VIII de coagulação - 250, 500 e 1000 UI & 5 & 0 & 12 & Estratégico \\
\hline Fator VIII p/ doença de von Willebrand - 450 a 500 UI & 3 & 0 & 4 & Estratégico \\
\hline Fator VIII recombinante $250 \mathrm{UI}, 500 \mathrm{UI}$ e $1.000 \mathrm{UI}$ & 2 & 1 & 5 & Estratégico \\
\hline Fator XIII de Coagulação - 250 UI & 1 & 0 & 1 & Estratégico \\
\hline Fibrinogênio (fator I) $-1 \mathrm{~g}$ & 1 & 0 & 1 & Estratégico \\
\hline Imunoglobulina antitetânica - 250 UI/ml & 2 & 0 & 2 & Estratégico \\
\hline Imunoglobulina hum. anti-hepatite B - 180-200 UI/ml & 2 & 0 & 1 & Estratégico \\
\hline Imunoglobulina humana antirrábica - $150 \mathrm{UI} / \mathrm{ml}$ & - & - & - & Estratégico \\
\hline Imunoglobulina hum. antivaricela zoster $125 \mathrm{UI} / 2,5 \mathrm{ml}$ & - & - & - & Estratégico \\
\hline Soro antiaracnídico (Loxosceles, Phoneutria e Tityus) & - & - & - & Estratégico \\
\hline Soro antibotrópico (pentavalente) & - & - & - & Estratégico \\
\hline Soro antibotrópico (pentavalente) e anticrotálico & - & - & - & Estratégico \\
\hline Soro antibotrópico (pentavalente) e antilaquético & - & - & - & Estratégico \\
\hline Soro antibotulínico AB (bivalente) & - & - & - & Estratégico \\
\hline Soro anticrotálico & - & - & - & Estratégico \\
\hline Soro antidiftérico & - & - & - & Estratégico \\
\hline Soro antielapídico (bivalente) & - & - & - & Estratégico \\
\hline Soro antiescorpiônico & - & - & - & Estratégico \\
\hline Soro antilonômico & - & - & - & Estratégico \\
\hline Soro antiloxoscélico (trivalente) & - & - & - & Estratégico \\
\hline Soro antirrábico & - & - & - & Estratégico \\
\hline Soro antitetânico & - & - & - & Estratégico \\
\hline Vacina adsorvida difteria e tétano adulto & - & - & - & Estratégico \\
\hline Vacina adsorvida difteria e tétano infantil & - & - & - & Estratégico \\
\hline Vacina adsorvida difteria, tétano e pertussis & - & - & - & Estratégico \\
\hline Vacina adsorvida difteria, tétano e pertussis (adulto) & - & - & - & Estratégico \\
\hline Vacina adsorvida difteria, tétano e pertussis (infantil) & - & - & - & Estratégico \\
\hline Vacina adsorvida difteria, tétano, pertussis, hepatite B & - & - & - & Estratégico \\
\hline Vacina adsorvida hepatite A (inativada) adulto & - & - & - & Estratégico \\
\hline Vacina adsorvida hepatite A (inativada) infantil & - & - & - & Estratégico \\
\hline
\end{tabular}


Vacina BCG

Vacina cólera (inativada)

Vacina febre amarela (atenuada)

Vacina febre tifoide (polissacarídica)

Vacina Haemophilus influenzae B (conjugada)

Vacina hepatite B (recombinante)

Vacina influenza trivalente (fragmentada, inativada)

Vacina meningocócica C (conjugada)

Vacina papilomavírus humano (recombinante)

Vacina pneumocócica 10-valente (conjugada)

Vacina pneumocócica 23-valente (polissacarídica)

Vacina poliomielite 1, 2 e 3 (inativada)

Vacina poliomielite 1 e 3 (atenuada)

Vacina raiva (inativada)

Vacina rotavírus humano G1P1 [8] (atenuada)

Vacina sarampo, caxumba, rubéola

Vacina sarampo, caxumba, rubéola e varicela (atenuada)

Vacina varicela (atenuada)

Adalimumabe - $40 \mathrm{mg}$

Alfadornase - $1 \mathrm{mg}$

Alfaepoetina - 1.000 - $10.000 \mathrm{UI}$

Alfainterferona $2 b-3-10.000 .000$ UI

Alfapeginterferona $2 \mathrm{a}-180 \mathrm{mcg}$

Alfapeginterferona $2 \mathrm{~b}-118,4 \mathrm{mcg}(80 \mathrm{mcg} / 0,5 \mathrm{~mL})$

Alfataliglicerase - $200 \mathrm{U}$

Alfavelaglicerase - $200 \mathrm{U}$ e $400 \mathrm{U}$

Betainterferona $1 \mathrm{a}-22 \mathrm{mcg}$

Betainterferona $1 \mathrm{~b}-9.600 .000 \mathrm{UI}(300 \mathrm{mcg})$

Certolizumabe Pegol - $200 \mathrm{mg} / \mathrm{mL}$

Etanercepte -25 e $50 \mathrm{mg}$

Filgrastim - $300 \mathrm{mcg}$

Golimumabe - $50 \mathrm{mg}$

Imunoglobulina humana - 0,5, 1, 2,5, 3, 5 e $6 \mathrm{~g}$

Imunoglobulina hum. anti-hepatite B - 100, 500 e 600UI

Infliximabe - $10 \mathrm{mg} / \mathrm{mL}$

Natalizumabe $-20 \mathrm{mg} / \mathrm{mL}$

Rituximabe $-10 \mathrm{mg} / \mathrm{mL}$

Somatropina - 4 e 12 UI

Tocilizumabe - $20 \mathrm{mg} / \mathrm{mL}$

Toxina botulínica A - 100 e $500 \mathrm{U}$
1

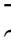

2

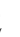

2

2

1

3

\begin{tabular}{|c|c|c|}
\hline 2 & 0 & Estratégico \\
\hline- & - & Estratégico \\
\hline 2 & 2 & Estratégico \\
\hline- & - & Estratégico \\
\hline- & - & Estratégico \\
\hline 0 & 2 & Estratégico \\
\hline 0 & 5 & Estratégico \\
\hline 1 & 6 & Estratégico \\
\hline 2 & 4 & Estratégico \\
\hline 0 & 4 & Estratégico \\
\hline 0 & 3 & Estratégico \\
\hline 0 & 3 & Estratégico \\
\hline 1 & 1 & Estratégico \\
\hline 0 & 2 & Estratégico \\
\hline 0 & 1 & Estratégico \\
\hline 1 & 10 & Estratégico \\
\hline 2 & 10 & Estratégico \\
\hline 0 & 5 & Estratégico \\
\hline 0 & 10 & Especializado \\
\hline 0 & 1 & Especializado \\
\hline 3 & 7 & Especializado \\
\hline 2 & 2 & Especializado \\
\hline 0 & 1 & Especializado \\
\hline 0 & 3 & Especializado \\
\hline 0 & 2 & Especializado \\
\hline 0 & 6 & Especializado \\
\hline 1 & 6 & Especializado \\
\hline 0 & 9 & Especializado \\
\hline 0 & 5 & Especializado \\
\hline 0 & 6 & Especializado \\
\hline 1 & 11 & Especializado \\
\hline 1 & 3 & Especializado \\
\hline 0 & 13 & Especializado \\
\hline 0 & 1 & Especializado \\
\hline 1 & 5 & Especializado \\
\hline 0 & 2 & Especializado \\
\hline 0 & 5 & Especializado \\
\hline 3 & 5 & Especializado \\
\hline 0 & 3 & Especializado \\
\hline 2 & 7 & Especializado \\
\hline
\end{tabular}

\section{DISCUSSÃO}

Os resultados indicam que todos os componentes da AF incluem medicamentos biológicos, presentes com maior frequência no componente estratégico e em seguida no componente especializado. Tal resultado pode ser explicado devido à presença das vacinas no componente estratégico e dos anticorpos monoclonais e interferon no componente especializado. Segundo Tanaka e Amorim (2014), grande parte dos produtos biotecnológicos está na categoria de medicamentos estratégicos, pois representam as tecnologias mais avançadas recentes para tratar acometimentos de alta gravidade e risco de morte e de grande impacto social. 
A maioria dos medicamentos biológicos presentes na RENAME-2017 que possuem registro ativo no país tem local de fabricação do medicamento em uma empresa que fica localizada no exterior, conforme podemos consultar através do portal da Anvisa, no item Consulta de Produtos Registrados, tendo poucas empresas nacionais que fabricam de fato estes produtos.

Observou-se também que, alguns medicamentos biológicos listados na RENAME não possuíam seus dados disponíveis para consulta na Consulta de assunto no portal da Anvisa na web. Isso pode deverse por uma divergência na nomenclatura entre a descrição do nome presente na lista da RENAME e a forma como é descrita o nome do medicamento no portal da Anvisa para consulta.

Para os medicamentos que possuem dados indisponíveis através da pesquisa no sítio eletrônico da Anvisa, a fim de encontrar dados de fabricação por outro meio, foi feita uma pesquisa com o nome do produto apresentado na RENAME no Google (www.google.com.br) para que fosse possível acrescentar estes dados ausentes.

Para a maioria destes medicamentos com informações indisponíveis, encontrou-se através da pesquisa supracitada dados que apresentam produção majoritariamente nacional. Por exemplo, o Instituto Butatan é responsável pela fabricação da maioria dos soros apresentados acima, junto a Fundação Ezequiel Dias, que também fabrica alguns outros soros. Importante também ressaltar que o Instituto Vital Brazil também possui fabricação de alguns soros, como antibotrópico, anticrotálico, antibotrópico-crotálico, antibotrópico-laquético, antiescorpiônico, antitetânico e antirrábico. Para estes produtos, considerando que sua produção é nacional, não existe dependência tecnológica e de mercado de outros países, o que traz maior autonomia para o Brasil, além de, possivelmente, estes produtos serem menos onerosos para a o governo, considerando não necessitar de importações, no mínimo, pois, para se afirmar de forma geral sobre os custos, um estudo sobre a carga tributária, custos de fabricação e importações.

Quando avaliadas as vacinas com informações ausentes, verificou-se que possuem perfil semelhante aos outros medicamentos listados que, conforme informações da Tabela 1 são apenas importados e podem até possuir etapas de embalagem em empresas nacionais. Estes são registrados localmente por empresas internacionais, as quais são apenas as detentoras de seu registro, porém a fabricação e, muitas vezes, embalagem primária e secundária são realizadas em empresas fora do país. Por exemplo, uma Betainterferona a primeira, comercializada no país é registrada e importada pela Fundação Oswaldo Cruz, mas fabricada por empresas internacionais, como podemos verificar através de consulta feita através do portal da Anvisa. Outros exemplos são alguns produtos comerciais dos anticorpos monoclonais Golimumabe, Natalizumabe, Rituximabe e Tocilizumabe que são registrados por empresas internacionais com filiais no Brasil, como Biogen e Roche, porém 
produzidos fora do país, fato que demonstra nossa dependência tecnológica de empresas internacionais na área de biológicos, já que ainda precisa importar a grande maioria destes medicamentos tão importante para a AF no país.

De acordo com Bermudez et al (2018), a questão da dependência tecnológica do Brasil no setor farmacêutico foi permanente ao longo do século XX e respondida em diferentes momentos, considerando o país na condição de "periferia" de um setor industrial que se consolidou principalmente nos países europeus e Estados Unidos, e cuja base dependeu do lançamento de inovações no mercado e na expansão de suas vendas.

No início da década de 1970, o estímulo à produção nacional de medicamentos esteve relacionado às ações de AF. Segundo Bermudez et al (2018), no final da década de 1990, com a Política Nacional de Medicamentos, iniciativas concretas voltadas ao setor industrial nacional pode ser observada, tanto pela aprovação da Política de Genéricos (Lei 9.787/99), quanto pelo Projeto Guarda Chuva, que assegurou financiamento aos laboratórios farmacêuticos oficiais (LFO), principalmente considerando a produção de medicamentos antirretrovirais no contexto da epidemia de HIV/aids. Já na segunda década com a aprovação da Política Industrial, Tecnológica e do Comércio Exterior (PITCE), o setor farmacêutico foi incluído, visando a redução da vulnerabilidade nacional, caracterizada por dependência externa nas áreas intensivas em tecnologia. A partir de 2008, na terceira década do SUS, o Complexo Industrial da Saúde (CIS) foi inserido entre os eixos estratégicos do planejamento em saúde do governo federal, resultando na aprovação de uma série de normas que deram novos contornos à política industrial, com ênfase nos esforços de retomada da indústria farmoquímica nacional e fortalecimento dos LFO. A partir de 2009, as Parcerias para Desenvolvimento Produtivo (PDP) foram estabelecidas como arranjos de transferência de tecnologia para fortalecer esses dois segmentos, considerando produtos adquiridos pelo SUS enquanto perspectiva de demanda constante, sem concorrência. Em que pese a previsão de uma política industrial enquanto enunciado e priorização governamental, diagnóstico recente dos LFO evidencia que aspectos da capacitação tecnológica e da capacidade em contribuir para o acesso a medicamentos pouco avançaram neste segmento, sugerindo baixa condição em responder às vulnerabilidades da $\mathrm{AF}$ no SUS. A seleção de tecnologias candidatas à produção local e ao desenvolvimento industrial deve ser considerada à luz da integralidade, o que, neste caso, implica analisar a dinâmica do mercado e priorizar aquelas sob risco de desabastecimento; áreas de vazios terapêuticos; e produtos de alto custo, com a finalidade de subsidiar a regulação de preços (Bermudez et al, 2018).

Em um estudo realizado por Reis et al. (2009), os Estados Unidos foram identificados como o principal produtor de medicamentos do mundo, respondendo por aproximadamente 39,3\% da 
produção global, seguido por Japão e Alemanha. Em conjunto, Estados Unidos, Europa e Japão possuíam 84\% de toda a produção desta classe de medicamentos. Em 2007 as 386 empresas de biotecnologia concentradas nos Estados Unidos foram responsáveis por um faturamento de cerca de 65 bilhões de dólares.

Apesar de sempre ter se destacado no mercado farmacêutico, concentrando algumas das empresas com maiores receitas, a Europa demorou um pouco a alavancar no segmento biotecnológico devido à relativa ausência de incentivos para criação de novas empresas de biotecnologia, reflexo do mercado de capital de riscos menos desenvolvido e do custo mais elevado da mão-de-obra qualificada nos países europeus. No entanto, a parcela europeia no mercado de biotecnológicos vem crescendo recentemente com a criação expressiva de novas empresas de biotecnologia e com o reposicionamento das grandes farmacêuticas de aquisição (REIS et al, 2009).

De acordo com Ernst \& Young (2007), notou-se um crescimento expressivo do mercado biotecnológico na Ásia. Na China estimou-se que o mercado de 12 bilhões de dólares, quadruplicasse em 10 anos. Na Índia, estimou-se que o mercado ultrapasse o dobro no mesmo período, passando de 6 bilhões para 15 bilhões de dólares. Esses dois países formam cerca de três vezes mais graduados em cursos relacionados à ciência e tecnologia, quando comparados aos Estados Unidos.

O desenvolvimento e a produção de medicamentos biológicos são fundamentais para a política de saúde brasileira, pois a demanda por aqueles que fazem parte da lista de medicamentos excepcionais representa uma parcela importante dos gastos do Sistema Único de Saúde (SUS) com medicamentos, devido ao seu alto valor. Além disso, a incorporação dos produtos biológicos no portfólio das empresas nacionais é importante para a manutenção da competitividade no médio e longo prazo, principalmente devido à perspectiva de aumento da concorrência (BENAVIDE, 2013).

Conforme ainda Tanaka e Amorim, 2014, no Brasil, a existência de um modelo de atenção à saúde, pautado pelo acesso universal associado às mudanças nos padrões demográficas e epidemiológicos tem ampliado consideravelmente a demanda por serviços de saúde, e pressionado os gastos públicos com medicamentos, principalmente no que se refere à incorporação de medicamentos produzidos por rota biotecnológica nas compras do SUS. Quando analisado, por exemplo, que do ponto de vista epidemiológico cresce o peso das doenças crônico-degenerativas (doenças do sistema circulatório, diabetes, doenças neurológicas e câncer) para as quais nem sempre há tratamentos curativos, compreendemos o potencial de consumo do Brasil.

A busca de competências nas novas plataformas da biotecnologia em saúde humana foi considerada estratégica pelo governo federal, objetivando o aumento da produção nacional de medicamentos biológicos (parcela significativa do déficit na balança comercial do complexo 
econômico industrial da saúde). A incorporação da rota biotecnológica por parte da indústria farmacêutica nacional apresentou também um elevado impacto econômico em função do potencial de mercado e do elevado valor agregado associado à produção biofarmacêutica. Nesse contexto, o ingresso na produção de medicamentos biológicos representa uma "janela de oportunidade" importante para o crescimento e consolidação da indústria de base química e biotecnológica no País. Portanto, o desenvolvimento do parque nacional foi visto pelo governo como uma oportunidade que deveria ser bem explorada pelas políticas públicas de fomento ao investimento na data da publicação do estudo (TANAKA e AMORIM, 2014).

$\mathrm{Na}$ perspectiva estratégica, para os laboratórios nacionais a produção de medicamento biotecnológico é crucial para a manutenção da competitividade no médio e longo prazo. Os produtos biotecnológicos já respondiam por uma parcela significante dos gastos do Ministério da Saúde com medicamentos. As instituições mais importantes na produção de medicamentos biológicos são o Instituto de Tecnologia em Imunológicos da Fiocruz (ligado ao Ministério da Saúde) e o Instituto Butantã (ligado à Secretaria de Saúde do Estado de São Paulo) (TANAKA E AMORIM, 2014).

Além disso, o Ministério da Saúde tem estabelecido parcerias para desenvolvimento produtivo; com essas parcerias, 29 produtos de saúde (28 medicamentos e o DIU) passarão a ser fabricados no País. Os medicamentos envolvem tratamento para doenças sexualmente transmissíveis, doenças crônicas não transmissíveis, doenças degenerativas, doença de Crohn, antipsicóticos, hemofilia e tuberculose (TANAKA E AMORIM, 2014).

Estão envolvidos 31 laboratórios, sendo 10 públicos e 21 privados, nacionais e estrangeiros. As parcerias supostamente gerariam uma economia estimada de R 400 milhões por ano. Este valor, somado à redução de custos gerada por inovação tecnológica e melhor gestão de recursos em vacinas, negociações e centralização de compras, supostamente levaria a uma redução de gastos equivalente a R \$ 1,7 bilhão por ano no orçamento do Ministério da Saúde (TANAKA E AMORIM, 2014).

Para estes autores, não obstante os impactos da recente crise financeira mundial em termos de pressões orçamentárias para cortes nos gastos públicos, o reconhecimento sobre a importância da contribuição econômica e social do setor biofarmacêutico tem levado diversos países a programar políticas e programas destinados a atrair para o território nacional as atividades de pesquisa e produção. Nos países desenvolvidos, providenciar o acesso aos medicamentos é crescentemente de responsabilidade governamental (exceto nos EUA).

Historicamente, as empresas multinacionais de base farmoquímica responderam pela maior parte da oferta, operando em mercados protegidos por barreiras à entrada e consumo privado. Na última década, dadas à expiração de patentes, aumento da competição com os produtores de 
medicamentos genéricos e das compras governamentais, a inovação do segmento percorreu a trilha dos fármacos de base biotecnológica (TANAKA E AMORIM, 2014).

O Brasil, cujo modelo de assistência farmacêutica aponta para o crescimento dos gastos governamentais e cuja população demandará cada vez mais medicamentos, o fomento à produção nacional e a determinação de regulação adequada à estratégia do complexo industrial da saúde (CIS) são condições para a ampliação do acesso dos cidadãos à saúde. (TANAKA E AMORIM, 2014)

Neste contexto, os produtos biológicos correspondem a uma parcela significativa dos gastos do SUS com a compra de medicamentos. Segundo análise feita por BENAVIDE (2013), seis dos medicamentos biológicos foram responsáveis pelo gasto de R 2 bilhões pelo Ministério da Saúde em 2011, sendo eles Humira (Adalimumabe), Herceptin (Transtuzumab), Remicade (Infliximabe), MabThera (Rituxan), Enbrel (Etanercept) e Avastin (Bevacizumab). Foi verificado que a maior parte dos medicamentos biológicos em destaque eram anticorpos monoclonais, e embora possuíssem mecanismos de ação diferenciados, a indicação terapêutica dominante era artrite reumatoide, seguida de câncer, o que nos permite evidenciar a importância do desenvolvimento e produção voltados para estas indicações. Em adição no ano de 2008, Reis et al (2010) verificaram que o SUS gastou 2,3 bilhões de reais com a compra de medicamentos de alto custo, contemplando 220 medicamentos destinados ao tratamento de 76 doenças de aproximadamente 730 mil usuários, ressaltando além da importância econômica, a importância deste grupo de medicamentos.

Na época da publicação do estudo de Benavide (2013), o Brasil tinha aproximadamente 2 milhões de portadores de artrite reumatoide e essa doença constituía o segundo maior motivo de incapacidade para o trabalho. Já o câncer, era responsável por cerca de 13,7\% das mortes registradas no país.

Espera-se que, se produzidos nacionalmente, o custo destes medicamentos possa cair em até 90\%. Uma plataforma de desenvolvimento de biossimilares também se mostraria estratégica, visto que as patentes têm expirado ano após ano (BENAVIDE, 2013).

Analisando a possível entrada da indústria farmacêutica nacional no campo biotecnológico através de experiências iniciais com biossimilares, observou-se que se trata de uma excelente e promissora oportunidade. A exemplo do que sucedeu após a Lei 9.787 de 1999 (política de Genéricos), que foi capaz de ampliar o acesso e reduzir custos tanto para o consumidor final quanto para o Ministério da Saúde, esperam-se que em função do vencimento das patentes de muitos medicamentos biológicos e atentas às margens de lucro que eles geram e o rápido crescimento desse mercado, muitas empresas farmacêuticas nacionais começassem a desenvolver biossimilares para entrar na briga por uma parcela desse mercado tão lucrativo (BENAVIDE, 2013). 
No Brasil já existem alguns medicamentos biológicos biossimilares já registados, como o Fiprima (filgrastim), o Remsima (infliximabe) e o Basaglar (insulina), por exemplo. Estes produtos podem ser considerados marcos nessa área, considerando que cada um deles foi pioneiro em seu respectivo campo, como primeiro biossimilar registrado pela via de desenvolvimento por comparabilidade, primeiro biossimilar totalmente fabricado no Brasil ou primeira insulina biossimilar do mercado brasileiro, demonstrando a dependência tecnológica que o nosso país ainda possui nesta área.

A importância dos produtos biológicos é reforçada pelo perfil epidemiológico brasileiro com tendência de maior prevalência de doenças crônico-degenerativas, como câncer, diabetes, doenças do sistema circulatório e doenças reumatológicas. Segundo Schramm et al (2004) esse grupo representava $66,3 \%$ da carga da doença no país.

\section{CONSIDERAÇÕES FINAIS}

Com base nas informações levantadas no presente estudo, podemos observar que os medicamentos biológicos são produtos de grande importância para a política de saúde brasileira, representando opções terapêuticas para inúmeras doenças, tendo presença significativa na RENAME, estando presente em todos os componentes, principalmente no estratégico.

Destes medicamentos todos possuem registro vigente no Brasil, sendo a estrutura de produção e desenvolvimento ainda bastante insipiente no Brasil que resulta em grande dependência tecnológica de outros países, além de grandes gastos por parte do governo, devido ao alto custo dos medicamentos. De modo geral, estes medicamentos são fabricados e/ou possuem alguma etapa do processo de fabricação principalmente em países europeus ou nos Estados Unidos e são importados e, por vezes, podem ter etapas de embalagem no país, mas são registrados por empresas internacionais, as quais são apenas as detentoras de seu registro, porém a fabricação e, muitas vezes, embalagem primária e secundária são realizadas em empresas fora do país. Este cenário só não é observado para alguns soros que possuem fabricação nacional principalmente pelo Instituto Butantan.

Desta forma, a fim de buscar independência dos mercados internacionais, o ideal seria que houvesse maior incentivo no desenvolvimento tecnológico nacional, ou até mesmo a transferência tecnológica completa, que impulsionaria a produção dos medicamentos biológicos em solo brasileiro, principalmente dos medicamentos essenciais, o que poderia trazer autonomia para o país, deixando mais eficiente a provisão de medicamentos para assistência farmacêutica aos pacientes que necessitam destes medicamentos e que não são produzidos no país. 
Além disso, parcerias com universidades para que sejam desenvolvida uma estrutura de pesquisa e desenvolvimento também pode contribuir para o crescimento do Brasil no campo dos medicamentos biológicos.

\section{REFERÊNCIAS}

BENAVIDE, V. G. Panorama sobre alguns entraves e desafios na produção nacional de biofármacos. Instituto Oswaldo Cruz. Rio de Janeiro, 2013.

BERMUDEZ, J. A. Z.; HASENCLEVER, L.; EPSZTEJN, R.; OLIVEIRA, M. O Acordo TRIPS da OMC e a Proteção Patentária no Brasil: Mudanças Recentes e Implicações para a Produção Local e o Acesso da População aos Medicamentos. Rio de Janeiro: Editora FIOCRUZ/ENSP, 2000. p. 131.

BERMUDEZ, J.A.Z. et al. Assistência Farmacêutica nos 30 anos do SUS na perspectiva da integralidade Ciência \& Saúde Coletiva, 23(6):1937-1951, 2018

BRAGA, TGP "Análise do Impacto de Estudos Clínicos no Registro de Produtos Biológicos no Brasil”. Brasília-DF, UnB, 2016. 106 f. Trabalho de Conclusão de Curso de Graduação em Farmácia, Universidade de Brasília.

BRASIL. PREÇOS MÁXIMOS DE MEDICAMENTOS POR PRINCÍPIO ATIVO, Câmara de Regulação do Mercado de Medicamentos- CMED - Anvisa. Atualizada em 19/11/2018.

BRASIL. Lei no 9.782, de 26 de janeiro de 1999. Define o Sistema Nacional de Vigilância Sanitária, cria a Agência Nacional de Vigilância Sanitária e dá outras providências. Diário Oficial da União, Brasília, DF, 27 de jan. 1999.

BRASIL. Portaria $n^{\circ} 354$, de 11 de agosto de 2006. Aprova e promulga o Regimento Interno da Agência Nacional de Vigilância Sanitária - ANVISA e da outras providências. Diário Oficial da União, Brasília, DF, 14 de ago. 2016.

BRASIL. ANVISA. Resolução - RDC no 55, de 16 de dezembro de 2010. Dispõe sobre o registro de produtos biológicos novos e produtos biológicos e dá outras providências. Publicado no Diário Oficial da União no 241, Brasília, DF, 17 dez. 2010.

BRASIL. Portaria n ${ }^{\circ} 107$, de 20 de setembro de 1994. Aprova as Normas para Análise de processo de Registro de Imunobiológicos, conforme Manual da Qualidade. Publicado em Diário Oficial da União , Brasília, DF, 1994.

BRASIL. ANVISA . Resolução - RDC nº 80, de 18 de março de 2002. Regulamento Técnico dos procedimentos de registro, de alteração e inclusão pós-registro e revalidação dos produtos biológicos. Publicado em Diário Oficial da União, Brasília, DF, 2002.

BRASIL. ANVISA . Resolução - RDC n 315, de 26 de outubro de 2005. Dispõe sobre o Regulamento Técnico de Registro, Alterações Pós-Registro e Revalidação de Registro dos Produtos Biológicos Terminados. Publicado em Diário Oficial da União, Brasília, DF, 2005.

BRASIL. ANVISA - Resolução No 49, de 20 de setembro de 2011. Dispõe sobre a realização de alterações e inclusões pós-registro, suspensão e reativação de fabricação e cancelamentos de registro de produtos biológicos e dá outras providências. Publicado no Diário Oficial da União $\mathrm{n}^{\circ} 183$. Brasília, DF, 22 set. 2011.

BRASIL. Relação nacional de medicamentos essenciais: Rename. Ministério da Saúde. Secretaria de Ciência, Tecnologia e Insumos Estratégicos. Brasília: Ministério da Saúde, 2017.

BRATS - Boletim Brasileiro de Avaliação em Tecnologia em Saúde. Medicamentos biológicos para o tratamento de artrite reumatoide. 2012;6(19):1-13.

CORDEIRO, A.M. et al. Revisão Sistemática: Uma Revisão Narrativa. Rev. Col. Bras. Cir. Vol. 34 No 6, Nov / Dez. 2007. 
ERNST \& YOUNG. Beyond Borders: Global Biotechnology Report 2007, 2007.

FEDERACIÓN LATINOAMERICANA DE LA INDUSTRIA FARMACÉUTICA Federación Latinoamericana de la Industria Farmacéutica. Biofármacos. (Federação Latino-americana da Indústria Farmacêutica). Março de 2006. P. 5-6

HU, X.; MA, Q.; ZHANG, S. Biopharmaceuticals in China. Biotechnol. J., 1, 1215-1224, 2006.

IMS HEALTH. The Global Use of Medicines : Outlook Through 2016, 2012

INTERFARMA- Associação da Indústria Farmacêutica de Pesquisa. Entendendo os medicamentos biológicos. 2012. Disponível em: http://www.interfarma.org.br/uploads/biblioteca/34-biologicossite.pdf. Acesso em: 16/06/2016.

MADEIRA, et al. Identificação de Biofármacos para Produção no Brasil. Fiocruz/UFRJ. 2013.

MORETO, L. D. Manual para registro de medicamentos biológicos no Brasil. Sindusfarma. São Paulo, 2015.

PARANHOS, J. et al. Um estudo da tramitação de patentes farmacêuticas concedidas pelo INPI póstrips. Gestion de la innovacion para la competitividade. Cidade do México. Altec 2016

PARANHOS, J. Projeto ABIA: Extensão das patentes e custos para o SUS. Associação Brasileira Interdisciplinar de Aids. 2016

RADER, R. A. What is a biopharmaceutical? Part 1: (Bio) Technology-based definitions, BioExecutive International, 60-65. 2005.

RAO, S. K. Pricing biologics: issues, strategic priorities and a conceptual model. J Commer Biotechnol., 17, 7-23, 2011.

REIS, C. et al. Biotecnologia para saúde humana: tecnologias, aplicações e inserção na indústria farmacêutica. BNDES Setorial, 29, p. 359-392, mar. 2009.

REIS, C.; PIERONE, J. P.; SOUZA, J. O. B. de, Biotecnologia para saúde no Brasil. BNDES Setorial, 32, p. 193-230, set. 2010.

REVERS, L.; FURCZON, E. An introduction to biologics and biosimilars. Part 1: biologics: what are they and where do they come from? Can Pharmacists J CPJ/RPC., 143, 134-139, 2010.

SAHOO, N.; CHOUDHURY, K.; MANCHKANTI, P., Manufacturing of biodrugs: need for harmonization in regulatory standards. Biodrugs., 23, 217-229, 2009.

SCHRAMM, Joyce Mendes de Andrade et al, Transição epidemiológica e o estudo de carga de doença no Brasil. Ciênc. Saúde coletiva, 9, 4, 897-908, 2004.

TANAKA, R. L., AMORIM, M. C. S, O mercado e as possibilidades da indústria de biofármacos no Brasil. Rev. Fac. Ciênc. Méd. Sorocaba, v. 16, n. 2, p. 86 - 92, 2014

WHO. Increasing access to HIV treatment in middle-income countries - Key data on prices, regulatory status, tariffs and the intellectual property situation. Switzerland: WHO, 2014 